\title{
Optimal shape of fibers in composite structure using Inverse variational principles
}

\author{
P. Procházka \\ Czech Technical University in Prague, Czech Republic
}

\begin{abstract}
Inverse variational principles proved their importance in shape optimization of structures. In this paper they are applied to searching for the optimal shape of fibers in a composite structure. As the boundary element method seems to be more promising than other modern numerical methods applied to the search for optimal shape, in the submitting paper the boundary element method is redefined to enable one to use such an approach, which leads to possibility for the optimal interfacial energies and, hence, to the optimal bearing capacity of the composite structure. Necessary discretization of the domain, which occurs in the finite elements, is suppressed in our case. Standard procedure in the finite elements leads to dependence of the stiffness matrix on the shape of the fibers. In this case, following a basic idea for homogenization and localization, concentration factors have to be calculated in terms of the boundary element method instead. These terms are dependent on the shape of the fibers. It appears that the procedure is still not convergent (we solve a strongly nonlinear problem) and additional constraint has to be involved in the formulation. In order to formulate and solve this problem, the idea of Inverse variational principles is applied here for expressing necessary quantities. The paper concentrates on the calculation of quantities, which are necessary to formulate the optimization problem. The main attention is focused on calculation of concentration factors, which play the most important role in the approach proposed.
\end{abstract}

Keywords: discrete element method, boundary element method, dynamical equilibrium. 


\section{Introduction}

Conventionally, the optimal shape design problem consists of minimization of an appropriate cost functional with certain constraints, such as equilibrium and compatibility conditions and design requirements. The formulation of the cost functional depends of the concrete intention of a designer. One of a reasonable and practical form of the cost functional respects the minimization of the strain energy of the body subjected to a specific load. Such a problem can easily be formulated in terms of inverse variational principles, which assure that the surface energy attains its minimum.

The inverse variational principles are naturally connected with finite element method, which starts with energetic formulation. But, the FEM is less suitable for the problems involving the problem like moving boundary, optimal shape, etc. On the other hand, the direct connection of the BEM with the variational principles is not seen at first sight (see [1]). In the latter paper optimization problems based on the inverse variational principles solved by boundary elements is formulated. This approach is extraordinarily advantageous, as no internal mesh has to be generated.

In [10] the optimal composite is obtained by using a two-step procedure: (i) first an ideal structure of the matrix material is found by weakening the polymer by an optimal arrangement of pores, and (ii) the rods are embedded in the matrix. The design parameters are the shape, volume fraction, and spatial arrangement of the piezoceramic rods, and the structure of the matrix material. It turns out that the optimal matrix is highly anisotropic and is characterized by negative Poisson's ratios in certain directions.

Since we are concentrated on optimization of composite structures using homogenization, the theory for periodic media given by Suquet [2] is used in this paper. Similarly to Suquet's examples symmetric problems are considered.

The way on how to formulate the problem starts with the idea of HashinShtrikman variational principles according to [3], which were worked out into integral form.

First, homogenization and localization, having the principal meaning in the approach introduced in this paper, is discussed using the boundary element method. Then the optimization of shape of fibers is formulated and solved using the information from the previous sections. Some examples are discussed at the end of this paper.

\section{Localization and homogenization of symmetric periodic structures}

Localization and homogenization is concisely described in Suquet [2]. Recall some basic consumption which we use later in the integral formulations. First, we denote quantities used in this text. Two different scales will naturally be introduced. The macroscopic scale, the homogeneous law in which is sought, will be described in coordinate system $\boldsymbol{x} \equiv\left\{x_{1}, x_{2}, x_{3}\right\}^{\mathrm{T}}$ and the microscopic 
scale - heterogeneous - is characterized in the system of coordinates $\boldsymbol{y} \equiv\left\{y_{1}, y_{2}, y_{3}\right\}^{\mathrm{T}}$. The medium is generally heterogeneous, but locally - in the microscopic scale - is assumed to be periodic, thus a representative volume element may be cut out from the structure and the periodicity conditions can be introduced on the boundary of this element.

Let us distinguish the quantities under study in dependence of the macroscopic or microscopic scale in the following manner: The displacements in the macroscopic level will be denoted as $\boldsymbol{U} \equiv\left\{U_{1}, U_{2}, U_{3}\right\}^{\mathrm{T}}$ while in the microscopic level as $\boldsymbol{u} \equiv\left\{u_{1}, u_{2}, u_{3}\right\}^{\mathrm{T}}$. Moreover, in macroscopic level, let us denote strains as $\boldsymbol{E} \equiv\left\{E_{i j}\right\}, i, j=1,2,3$ and stresses as $\boldsymbol{S} \equiv\left\{S_{i j}\right\}, i, j=1,2,3$. In the microscopic level let us denote strains as $\varepsilon \equiv\left\{\varepsilon_{i j}\right\}, i, j=1,2,3$ and stress as $\boldsymbol{\sigma} \equiv\left\{\sigma_{i j}\right\}, i, j=1,2,3$. Define also the microscopic-macroscopic relation of the averaged stresses and strains by

$$
S_{i j}=\frac{1}{\operatorname{meas} \Omega} \int_{\Omega} \sigma_{i j} \mathrm{~d} \Omega=<\sigma_{i j}>, \quad E_{i j}=\frac{1}{\operatorname{meas} \Omega} \int_{\Omega} \varepsilon_{i j} \mathrm{~d} \Omega=<\varepsilon_{i j}>
$$

where $<$. $>$ stands for the average, $\Omega$ is the representative volume element, and meas $\Omega$ is its volume, $\Omega=\Omega^{\mathrm{f}} \cup \Omega^{\mathrm{m}}, \Omega^{\mathrm{f}} \cap \Omega^{\mathrm{m}}=0, \Omega^{\mathrm{f}}$ denotes the domain of fiber and $\Omega^{\mathrm{m}}$ is the domain of matrix. As usual, meas $\Omega$ is set to unity. Note that average usually means homogenization, but one should use that term with care: there are many kinds of averaging.

The elasticity system (equilibrium equations, kinematical conditions and Hooke's law) is defined as (small deformation theory is imposed):

$$
\operatorname{div} \boldsymbol{\sigma}(\boldsymbol{y})=0, \quad \boldsymbol{\sigma}(\boldsymbol{y})=\boldsymbol{L}(\boldsymbol{y}): \boldsymbol{\varepsilon}(\boldsymbol{y}), \quad \boldsymbol{\varepsilon}=\frac{1}{2}\left(\nabla \boldsymbol{u}+\nabla^{\mathrm{T}} \boldsymbol{u}\right) \quad \text { in } \Omega
$$

and periodic boundary conditions along the boundary of the unit cell $\partial \Omega$ are given.

Localization consists of the solution of system of elasticity system (equilibrium equations, kinematical conditions and Hooke's law) on the representative volume element (or unit cell) for concentration factors $\boldsymbol{A}^{\mathrm{f}}$ of fibers and $\boldsymbol{A}^{\mathrm{m}}$ for matrix:

$$
\begin{array}{ll}
\varepsilon_{i j}^{\mathrm{f}}(\boldsymbol{u}(\boldsymbol{y}))=A_{i j k l}^{\mathrm{f}}(\boldsymbol{y}) E_{k l}, & \boldsymbol{y} \in \Omega^{\mathrm{f}} \\
\varepsilon_{i j}^{\mathrm{m}}(\boldsymbol{u}(\boldsymbol{y}))=A_{i j k l}^{\mathrm{m}}(\boldsymbol{y}) E_{k l}, & \boldsymbol{y} \in \Omega^{\mathrm{m}}
\end{array}
$$

Periodic boundary conditions will be employed on $\partial \Omega$. If $\boldsymbol{n} \equiv\left\{n_{1}, n_{2}, n_{3}\right\}$ is outward unit normal to $\partial \Omega$, it holds: 
stress: components of tractions $p_{i}=\sigma_{i j} n_{j}$ are opposite on the opposite sides, strains: the local strain tensor $\boldsymbol{\varepsilon}(\boldsymbol{u})$ is split into its average $\boldsymbol{E}$ and a fluctuating term $\overline{\boldsymbol{\varepsilon}}(\boldsymbol{u})$ as:

$$
\boldsymbol{\varepsilon}(\boldsymbol{u})=\boldsymbol{E}+\boldsymbol{\varepsilon}^{*}(\boldsymbol{u}), \quad \boldsymbol{\varepsilon}^{*}(\boldsymbol{u})=\boldsymbol{\varepsilon}\left(\boldsymbol{u}^{*}\right), \quad<\boldsymbol{\varepsilon}\left(\boldsymbol{u}^{*}\right)>=0
$$

displacements: $u_{i}^{*}$ are the same at opposite sides. Hence, the fluctuating displacement $\boldsymbol{u}^{*}$ may be considered a periodic field, up to a rigid displacement that will be disregarded. The geometry and denotation is obvious from Fig. 1 for $2 \mathrm{D}$ case. Interfacial surface between fiber and matrix is denoted by $\Gamma$.

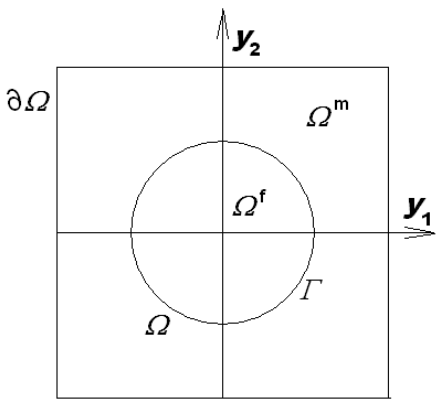

Figure 1: $\quad$ Unit cell used in the study.

As we concentrate on symmetric problems, and linear elasticity is considered (hence superposition is admitted), the periodicity conditions can be substituted according to Figs. 3, 4, 5, where only first quarter is considered with different boundary conditions, describing symmetry or antisymmetry of particular problems.

In Fig. 2 the geometry, supports and loading for the response of $E_{11}$ is depicted, in Fig. 3 the same for $E_{22}$ and in Fig. 4 for $E_{12}$. For the sake of simplicity two dimensional case is drawn. The triangles denoting supports are rollers.

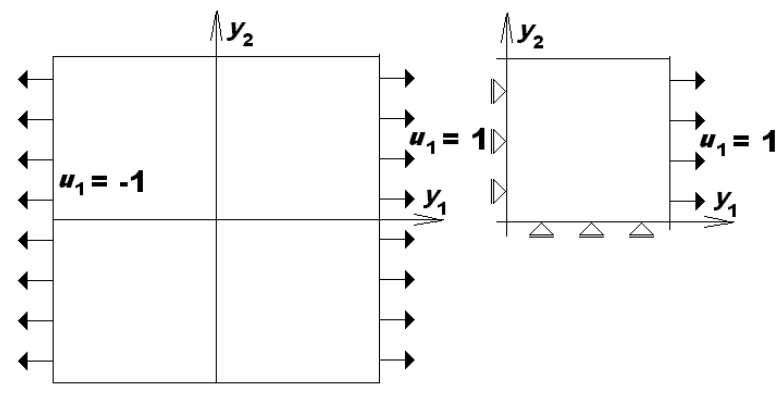

Figure 2: $\quad$ Original and computational model for responses of $E_{11}$. 


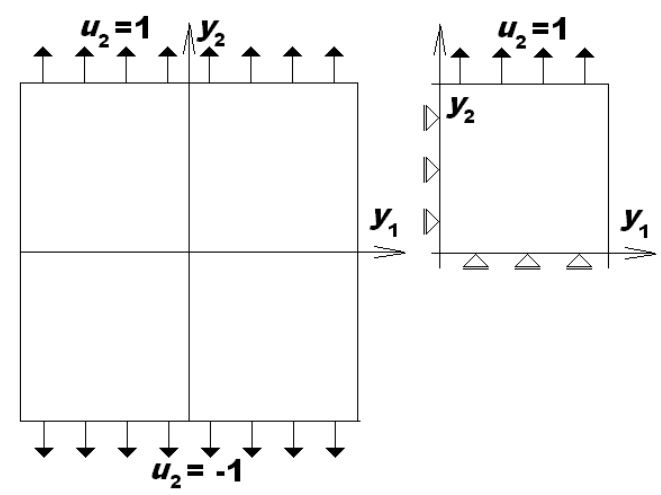

Figure 3: $\quad$ Original and computational model for responses of $E_{22}$.

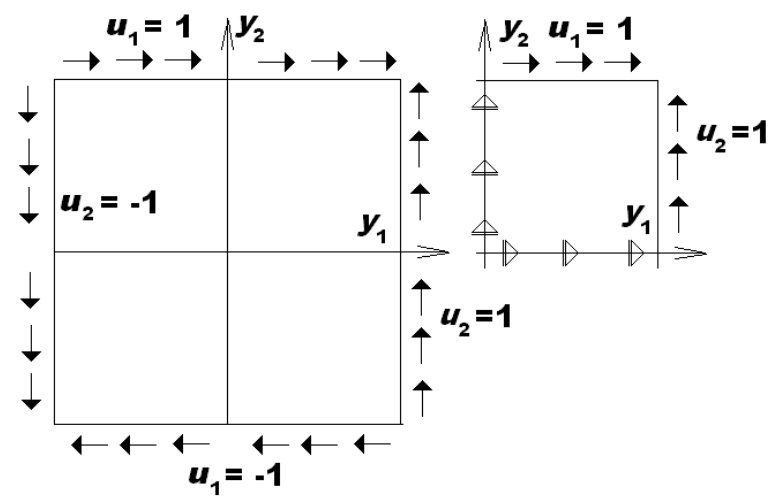

Figure 4: Original and computational model for responses of $E_{12}$.

Under the above described circumstances Hill's energy condition holds valid, as proved, e.g., by Suquet, [2]:

$$
<\sigma_{i j}(y) \varepsilon_{i j}(y)>=S_{i j} E_{i j}
$$

Using (1), $\left(2_{2}\right)$ and (3) the components of the overall stresses are written in the following way:

$$
S_{i j}=<\sigma_{i j}(\boldsymbol{y})>=<L_{i j k l}(\boldsymbol{y}) \varepsilon_{k l}(\boldsymbol{y})>=\left(<L_{i j k l}^{\mathrm{f}} A_{k l \alpha \beta}^{\mathrm{f}}(\boldsymbol{y})>_{\mathrm{f}}+<L_{i j k l}^{\mathrm{m}} A_{k l \alpha \beta}^{\mathrm{m}}(\boldsymbol{y})>_{\mathrm{m}}\right) E_{\alpha \beta}
$$

where $\langle.\rangle_{\mathrm{f}}$ stands for average on fiber and $\langle.\rangle_{\mathrm{m}}$ is the average on matrix. This averaging process is made in such a way that the integrals are taken over fiber and matrix, respectively, but the denominator generally remains meas $\Omega$, see (2). 
By definition, the homogenized stiffness matrix $\boldsymbol{L}^{*}$ is written as:

$$
S_{i j}=L_{i j k l}^{*} E_{k l}
$$

Comparing (6) and (7) the overall stiffness matrix follows as

$$
L_{i j k l}^{*}=<L_{i j k l}^{\mathrm{f}} A_{k l \alpha \beta}^{\mathrm{f}}(\boldsymbol{y})>_{\mathrm{f}}+<L_{i j k l}^{\mathrm{m}} A_{k l \alpha \beta}^{\mathrm{m}}(\boldsymbol{y})>_{\mathrm{m}}
$$

It is worth noting that the homogenized stiffness matrix is symmetric with similar properties as that of the classical stiffness matrix in the problem defined in the microscale.

\section{Localization using BEM}

Without lack of generality, let us consider a symmetric unit cell depicted in Fig. 1, for example. The overall strain $E_{i j}$ is assumed to be given independently of the shape of the unit cell and of the shape of the fiber. The loading of this unit cell will be given by unit impulses of $E_{i j}$, i.e. we successively select $E_{i_{0} j_{0}}=E_{j_{0} i_{0}}=1 ; E_{i j}=0$ for either $i_{0} \neq i$ or $j_{0} \neq j$. How to select the unit impulses of the overall strain components will be discussed later on.

Now we concentrate our attention on the approach of computing the concentration factors, which play the most important role in our solution of the optimal problem.

First let us specify the boundary conditions, being equivalent to the unit impulses of the overall strain components. In elasticity it is possible to prescribe the overall strain field all over the domain $\Omega$ of the unit cell. Then the solution of responses to the unit impulses is given by the periodicity conditions and tractions along the interfacial surface $\Gamma$ between fibers and matrix. This approach is little bit cumbersome in case of debonding is admitted. The latter case is not considered here, but we apply more general form of introducing the unit impulses. It is well known that because of identity, see $\left(2_{3}\right)$, and Green's theorem it holds:

$$
\begin{aligned}
E_{i j}=\int_{\Omega} \varepsilon_{i j}(\boldsymbol{y}) \mathrm{d} \Omega=\frac{1}{2} \int_{\Omega}\left[\frac{\partial u_{i}}{\partial y_{j}}\right. & \left.(\boldsymbol{y})+\frac{\partial u_{j}}{\partial y_{i}}(\boldsymbol{y})\right] \mathrm{d} \Omega= \\
= & -\frac{1}{2} \int_{\partial \Omega}\left[u_{i}(\boldsymbol{y}) n_{j}(\boldsymbol{y})+u_{j}(\boldsymbol{y}) n_{i}(\boldsymbol{y})\right] \mathrm{d} \gamma(\boldsymbol{y})
\end{aligned}
$$

From (9) it immediately follows that the unit responses are given by prescribed displacements along the boundary of the unit cell. Moreover, using symmetry assumed in the beginning of this paper, we can solve the problem only on one 
quarter of the unit cell and get for the normal components of strains boundary conditions of the first quarter by Fig. 2 and Fig. 3 and for the shear strains boundary conditions according to Fig. 4 .

The procedure is split into two steps. Assume the above described surface displacements to be prescribed along the entire boundary $\partial \Omega$ and there are no body forces here. In the first step, the unit cell obeys static equilibrium equations and linear homogeneous Hooke's law (homogeneous and isotropic medium):

$$
\begin{gathered}
\sigma_{i j}^{0}=L_{i j k l}^{0} \varepsilon_{k l}^{0}, \text { in } \Omega, \quad \text { boundary conditions are fulfilled on } \partial \Omega \\
\text { in the sense of individual states }
\end{gathered}
$$

$L_{i j k l}^{0}$ are components of not yet determined material stiffness matrix (stiffness tensor). These components will be stated later. Such a medium is called comparative one.

The solution of (10) is easy, as the comparative medium is homogeneous and isotropic:

$$
u_{i}^{0}=E_{i j} y_{j}, \quad \varepsilon_{i j}^{0}=E_{i j} \quad \text { in } \Omega, \quad p_{i}^{0}=\sigma_{i j}^{0} n_{j}=L_{i j k l}^{0} E_{k l} n_{j} \quad \text { on } \partial \Omega
$$

In the second step a geometrically identical unit cell is considered. Also the loading and boundary conditions on $\partial \Omega$ remain valid. Define

$$
\bar{u}_{i}=u_{i}-u_{i}^{0}=u_{i}=E_{i j} y_{j}, \bar{\varepsilon}_{i j}=\varepsilon_{i j}-\varepsilon_{i j}^{0}=\varepsilon_{i j}-E_{i j}, \bar{\sigma}_{i j}=\sigma_{i j}-\sigma_{i j}^{0}=\sigma_{i j}-L_{i j k l}^{0} E_{k l}
$$

Our next aim is to determine primed quantities, components of displacement vector $\bar{u}_{i}$ and components of strain and stress tensors $\bar{\varepsilon}_{i j}$ and $\bar{\sigma}_{i j}$. In order to do so, system of fifteen equations of elasticity (2) has to be formulated for the primed set. We start with Hooke's law, which is valid for heterogeneous medium:

$$
\sigma_{i j}(\boldsymbol{y})=L_{i j k l}(\boldsymbol{y}) \varepsilon_{k l}(\boldsymbol{y}) \quad \text { in } \Omega
$$

Since the material stiffness tensor appears to be nonhomogeneous and unisotropic, idea used in [3], among others, will be adapted also here:

$$
\sigma_{i j}(\boldsymbol{y})=L_{i j k l}^{0} \varepsilon_{k l}(\boldsymbol{y})+\tau_{i j}(\boldsymbol{y}) \quad \text { in } \Omega
$$

where $\tau_{i j}$ are components of polarization tensor and the direct relation between stresses and strains becomes homogeneous and isotropic, so that integral formulation of elastic problem may be formulated. Subtracting (13) and (12) yields: 
142 Computer Methods and Experimental Measurements VIII

$$
\tau_{i j}=\left[L_{i j k l}\right] \varepsilon_{k l}, \quad\left[L_{i j k l}\right]=L_{i j k l}-L_{i j k l}^{0}
$$

which can be considered a definition of polarization tensor. Moreover, transformation to the primed system will not disturb the direct relation stresses strains, as after substituting $(13)$ to $\left(11_{3}\right)$ gives:

$$
\bar{\sigma}_{i j}=\sigma_{i j}-\sigma_{i j}^{0}=L_{i j k l}^{0} \varepsilon_{k l}+\tau_{i j}-\sigma_{i j}^{0}=L_{i j k l}^{0} \varepsilon_{k l}+\tau_{i j}-L_{i j k l}^{0} E_{k l}=L_{i j k l}^{0} \bar{\varepsilon}_{k l}+\tau_{i j}
$$

Since both $\sigma_{i j}$ and $\sigma_{\mathrm{i} j}^{0}$ are statically admissible, it holds (the following equations must be defined in the sense of distributions):

$$
\frac{\partial\left(L_{i j k l}^{0} \bar{\varepsilon}_{k l}+\tau_{i j}\right)}{\partial y_{j}}=0 \quad \text { in } \Omega, \quad \bar{u}_{i}=u_{i}-u_{i}^{0}, \quad \bar{p}_{i}=p_{i}-p_{i}^{0} \quad \text { on } \partial \Omega
$$

Following the assumption of the same prescribed boundary conditions, some of the terms in (16) disappear.

Owing to constant distribution of $L_{i j k l}^{0}$ in $\Omega$, the equivalent integral formulation can be written as:

$$
\begin{aligned}
& \bar{u}_{m}(\boldsymbol{\xi})=\int_{\partial \Omega} p_{m i}^{*}(\boldsymbol{y}, \boldsymbol{\xi}) \bar{u}_{i}(\boldsymbol{y}) \mathrm{d} \gamma(\boldsymbol{y})-\int_{\partial \Omega} u_{m i}^{*}(\boldsymbol{y}, \boldsymbol{\xi}) \bar{p}_{i}(\boldsymbol{y}) \mathrm{d} \gamma(\boldsymbol{y})+ \\
& +\left(\left[L_{i j k l}^{\mathrm{f}}-L_{i j k l}^{0}\right] \int_{\Omega^{\mathrm{f}}}+\left[L_{i j k l}^{\mathrm{m}}-L_{i j k l}^{0}\right] \int_{\Omega^{\mathrm{m}}}\right)_{\varepsilon_{m i j}^{*}}^{*}(\boldsymbol{y}, \boldsymbol{\xi}) \bar{\varepsilon}_{k}(\boldsymbol{y}) \mathrm{d} \Omega{ }^{\boldsymbol{\xi} \in \partial \Omega} \\
& c_{m n}(\boldsymbol{\xi}) \bar{u}_{n}(\boldsymbol{\xi})=\int_{\partial \Omega} p_{m i}^{*}(\boldsymbol{y}, \boldsymbol{\xi}) \bar{u}_{i}(\boldsymbol{y}) \mathrm{d} \gamma(\boldsymbol{y})-\int_{\partial \Omega} u_{m i}^{*}(\boldsymbol{y}, \boldsymbol{\xi}) \bar{p}_{i}(\boldsymbol{y}) \mathrm{d} \gamma(\boldsymbol{y})+ \\
& +\left(\left[L_{i j k l}^{\mathrm{f}}-L_{i j k l}^{0}\right] \int_{\Omega^{\mathrm{f}}}+\left[L_{i j k l}^{\mathrm{m}}-L_{i j k l}^{0}\right] \int_{\Omega^{\mathrm{m}}} \sigma_{m i j}^{*}(\boldsymbol{y}, \boldsymbol{\xi}) \bar{\varepsilon}_{k}(\boldsymbol{y}) \mathrm{d} \Omega{ }^{\boldsymbol{\xi} \in \partial \Omega}\right.
\end{aligned}
$$

where $c_{m n}$ are components of a tensor depending on position $\xi \in \partial \Omega$ and the quantities with asterisks are given kernels.

Differentiating (17) by $\xi_{n}$, applying Hooke's law, $L_{m n k l}^{\mathrm{f}}=L_{n m k l}^{0}$ and putting $\boldsymbol{\xi} \in \partial \Omega$ provides

$$
\begin{aligned}
& \bar{\varepsilon}_{m n}(\boldsymbol{\xi})=\int_{\partial \Omega} P_{n m i}^{*}(\boldsymbol{y}, \boldsymbol{\xi}) \bar{u}_{i}(\boldsymbol{y}) \mathrm{d} \gamma(\boldsymbol{y})-\int_{\partial \Omega} U_{n m i}^{*}(\boldsymbol{y}, \boldsymbol{\xi}) \bar{p}_{i}(\boldsymbol{y}) \mathrm{d} \gamma(\boldsymbol{y})+ \\
& +\int_{\Omega^{\mathrm{m}}}\left(\left[L_{m n k l}^{\mathrm{m}}-L_{m n k l}^{0}\right]\right) \Sigma_{n m i j}^{*}(\boldsymbol{y}, \boldsymbol{\xi}) \bar{\varepsilon}_{k l}(\boldsymbol{y}) \mathrm{d} \Omega
\end{aligned}
$$


and after discretization of the above equation relation (3) can easily be obtained. As sum of concentration factors on domain $\Omega$ is unity tensor, we can write:

$$
L_{i j k l}^{*}=<L_{i j k l}^{\mathrm{f}} A_{k l \alpha \beta}^{\mathrm{f}}(y)>_{\mathrm{f}}+<L_{i j k l}^{\mathrm{m}} A_{k l \alpha \beta}^{\mathrm{m}}(\boldsymbol{y})>_{\mathrm{m}}
$$

and the energy functional is formulated as, see, e.g., [1]:

$$
\Pi\left(\boldsymbol{u}, \Omega^{\mathrm{f}}\right)=\frac{1}{2}\left[L_{i j k l}^{\mathrm{f}}<A_{k l \alpha \beta}^{\mathrm{f}}\left(p_{s}\right)>_{\mathrm{f}}+L_{i j k l}^{\mathrm{m}}<A_{k l \alpha \beta}^{\mathrm{m}}\left(p_{s}\right)>_{\mathrm{m}}\right] E_{i j} E_{\alpha \beta}-\lambda\left(\int_{\Omega^{\mathrm{f}}} d \Omega-C\right)
$$

In the sense of the Inverse variational principle the lagrangian multiplier remains the same along the interfacial boundary, which provides algorithm described in [4].

\section{Example}

Unit cell is considered with fiber volume ratio equal to $0.21(\pi / 16)$. Since we compare energy densities at nodal points of the interfacial boundary, the relative energy density $\lambda$ may be regarded as the comparative quantity influencing the movement of the interfacial boundary. One phase possesses the following material properties: Young's modulus of the first one is $E^{1}=210 \mathrm{MPa}$, Poisson's ratio $v^{1}=0.16$; and the second $E^{2}=17 \mathrm{MPa}$, and $v^{2}=0.3$.

In Fig. 5 the starting shape and the final, optimal shape are depicted for stiff fiber (phase 1) and weaker matrix (phase 2). In Fig. 6 similar picture is presented for weak fiber (phase 2) and stiffer matrix (phase 1). In both cases, the stiffer phase "tries" to occupy larger area exposed to loading.
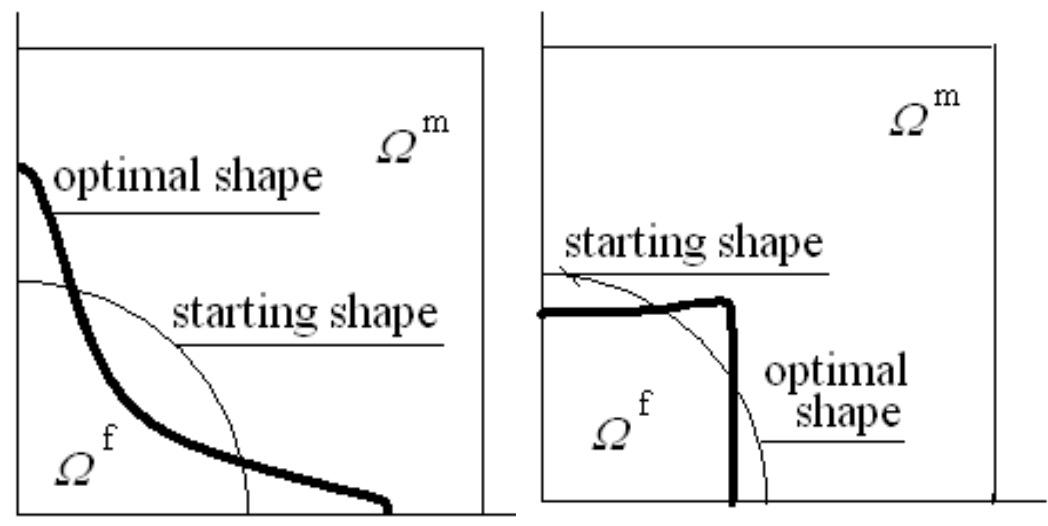

Figure 5: Optimal shapes for stiff and weak fibers. 


\section{Conclusions}

In this paper inverse variational principle has been applied to the solution of optimal fiber shape design on a unit cell of periodic composite structure. When searching for optimal shape design of fibers in composite structures, many formulations have been used in the past. They very often start with minimum strain energy function. This assumption is in Inverse variational principles fulfilled implicitly. A natural requirement is the restriction to the constant volume or area in $2 \mathrm{D}$ or volume in $3 \mathrm{D}$. Periodic distribution of fibers is considered in this paper.

The requirement of the constant volume or area seams to be restrictive, particularly when expecting application of Inverse variational principles to larger range of problems. Actually, it is not so. The constant $C$ may change, too. Thus the formulation has to be extended in such a way that $C$ is involved into the problem as a new variable and may be variated (differentiated) in some reasonable way.

\section{Acknowledgement}

The financial support of Grant agency of the Czech Republic, project No. $103 / 07 / 0304$ is greatly appreciated.

\section{References}

[1] Prochazka, P., Shape optimal design using Inverse Variational Principles, submitted to EABE

[2] Suquet P.M., Elements of homogenization for inelastic solid mechanics, Lecture Notes in Physics, 272 - Homogenization Technique for Composite Media, 1987

[3] Prochazka P., Sejnoha, J., Behavior of composites on bounded domain. BE Communications, 7, 1, 6-8, 1996

[4] Prochazka, P., Shape optimization of composites based on minimum potential energy, OPTI 2007, New Forest, UK 